\title{
CHARACTERIZATION OF THE SMOS INSTRUMENTAL ERROR PATTERN CORRECTION OVER THE OCEAN
}

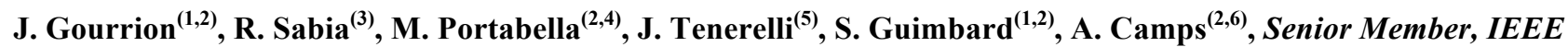 \\ (1) Institut de Ciències del Mar (ICM-CSIC), Barcelona, Spain. \\ (2) SMOS Barcelona Expert Centre (SMOS-BEC), Barcelona, Spain. \\ (3) European Space Agency (ESA-ESRIN), Frascati, Italy. \\ (4) Unitat de Tecnologia Marina (UTM-CSIC), Barcelona, Spain. \\ (5) Collecte Localisation Satellites (CLS), Plouzané, France. \\ ${ }^{(6)}$ Remote Sensing Laboratory, Universitat Politècnica de Catalunya and IEEC CRAE/UPC, Barcelona, Spain.
}

\section{Email: gourrion@icm.csic.es}

\begin{abstract}
The Soil Moisture and Ocean Salinity (SMOS) mission was launched on November $2^{\text {nd }}, 2009$ aiming at providing sea surface salinity (SSS) estimates over the oceans with frequent temporal coverage. The detection and mitigation of residual instrumental systematic errors in the measured brightness temperatures is a key step prior to the SSS retrieval. For such purpose, the socalled Ocean Target Transformation (OTT) technique is currently used in the SMOS operational SSS processor. In this study, an assessment of the OTT is performed. It is found that, to compute a consistent and robust OTT, a large ensemble of measurements is required. Moreover, several effects are reported to significantly impact the OTT computation, namely, the apparent instrument (temporal) drift, forward model imperfections, auxiliary data (used by forward model) uncertainty and external error sources, such as galactic noise and Sun effects (among others). These effects have to be properly mitigated or filtered during the OTT computation, so as to successfully retrieve SSS from SMOS measurements.
\end{abstract}

\section{INTRODUCTION}

The European Space Agency (ESA) Soil Moisture and Ocean Salinity (SMOS) Earth Explorer opportunity mission was launched on November $2^{\text {nd }}, 2009$. Over the oceans, it provides synoptic sea surface salinity (SSS) measurements with spatial and temporal coverage adequate for large-scale oceanographic studies [1]. The single payload onboard SMOS is the Microwave Imaging Radiometer using Aperture Synthesis (MIRAS), a novel fully-polarimetric L-band radiometer which measures the brightness temperature $\left(T_{B}\right.$, Level 1) by means of 2-D aperture synthesis interferometry [2]. To allow proper SSS retrievals in a single satellite overpass (Level 2 product) from the multi-angular $T_{B} S$ measured by MIRAS, a comprehensive inversion scheme has been defined and implemented in the operational retrieval processing chain, i.e., the Level 2 Ocean Salinity processor (L2OS) [3], despite several issues which still remain critical. The prescribed accuracy of SMOS SSS data is set to $1.2 \mathrm{psu}$ (practical salinity unit) at Level 2 and 0.1 psu at Level 3, i.e., after averaging in a 10 - or 30 -day period and $2^{\circ} \times 2^{\circ}$ or $1^{\circ} \times 1^{\circ}$ spatial boxes, respectively [1].

After launch, the SMOS satellite has undergone the InOrbit Commissioning Phase (IOCP), the customary 6month calibration and checkout period. Despite the substantial progress in the instrument calibration, improvement on the detection of instrument and image reconstruction $\mathrm{T}_{\mathrm{B}}$ biases over the oceans remains critical. In fact, systematic antenna-based error patterns have been detected in SMOS $\mathrm{T}_{\mathrm{B}}$ measurements at X-pol $\left(\mathrm{T}_{\mathrm{XX}}\right)$, Y-pol $\left(\mathrm{T}_{\mathrm{YY}}\right)$, and Stokes' third and fourth parameters, as already foreseen in [4]. These error patterns have typical amplitude of $\pm 5 \mathrm{~K}$ (see Fig. 1) [5]. They have different possible sources. While antenna pattern misestimates for the different receivers are supposed to be mitigated by the Flat Target Transformation (FTT), residual biases may still be present due to imperfection of this methodology related to the angular variation of the ocean $\mathrm{T}_{\mathrm{B}}$. Furthermore, the image reconstruction procedure itself and calibration residual errors may be partly responsible for the reported systematic patterns. These are estimated and mitigated using the so-called Ocean Target Transformation (OTT) [6] technique. The quality of the retrieved geophysical parameters strongly improves when the OTT is applied [5] while much work is still required to improve the estimation of these systematic patterns.

An intrinsic assumption of this approach is that these instrumental errors are additive (in $\mathrm{T}_{\mathrm{B}}$ space) and systematic. As such, the OTT is expected to be stable (invariant) in time. Nevertheless, imperfections in instrument calibration, data processing, OTT formalism, external noise sources (Sun, galaxy, etc.) introduce variability in the images, misleading the systematic instrumental pattern estimation. OTT inaccuracies propagate down to the retrieved salinities and, as such, are very important to assess and reduce.

Aiming at discriminating the potential sources of variability (i.e., error) of the OTT, three different effects have been examined in this study, with the general focus of devising possible improvements in the overall bias 
mitigation strategy. These effects are: the number of measurements used to compute the OTT and its temporal and spatial variability. In Section 2, the OTT technique is introduced. In Section 3, the data and the filtering criteria are presented. The analysis of the aforementioned potential sources of error is performed in Section 4. Finally, the concluding remarks can be found in Section 5.

\section{THE OCEAN TARGET TRANSFORMATION}

To account for instrument- and reconstruction-related systematic errors, the OTT estimates the error in antenna coordinates $(\xi, \eta)$ over a large number of snapshots (i.e., SMOS reconstructed images) as the average misfit between the $\operatorname{SMOS}\left(T_{B}^{S M O S}\right)$ and the modeled $\left(T_{B}^{\text {model }}\right)$ brightness temperatures, in the following way:

$$
\operatorname{OTT}(\xi, \eta)=\left\langle\left(T_{B}^{S M O S}(\xi, \eta)-T_{B}^{\text {model }}(\xi, \eta)\right)\right\rangle
$$

Several forward models relating the geophysical conditions such as Sea Surface Temperature (SST), salinity, and wind speed with the expected measurements are used to convert the auxiliary information into $T_{B}^{\text {model }}$. Both theoretical and empirically-based formulations have been derived prior to the SMOS launch (see [1] for detailed references) and are currently used in the L2OS. The resulting spatial pattern is subtracted from the SMOS measurements prior to SSS retrieval. Figure 1 displays a sample OTT for $\mathrm{T}_{\mathrm{XX}}$ and $\mathrm{T}_{\mathrm{YY}}$ over the Alias-Free Field of View (AFFOV), both calculated from a 16-day filtered dataset over the period August $3^{\text {rd }}$ to $18^{\text {th }}$ (see section 4.1).

The OTT currently used in the L2OS [3] is based on (1) and derived with data from a single half-orbit acquired on August $3^{\text {rd }}, 2010$ over the Pacific Ocean.
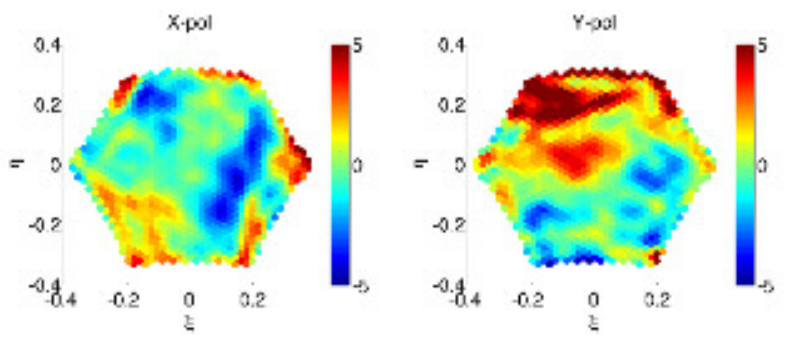

Fig. 1 Sample OTT-derived spatial pattern in Kelvin for a filtered dataset over the period August $3^{\text {rd }}$ to $18^{\text {th }}$ for $T_{X X}$ (left) and $T_{Y Y}$ (right).

\section{DATA FILTERING}

As a pre-requisite for a proper characterization of the OTT, a robust dataset has to be built. This is done by developing a well-suited data filtering strategy. A total of 48 days of SMOS L1B data, i.e., $\mathrm{T}_{\mathrm{B}}$ measurements at the antenna reference frame, collected from the SMOS
Data Processing Ground Segment (DPGS) have been used. In particular, the dataset corresponds to the period immediately after the updated FTT calibration, performed on August $3^{\text {rd }}, 2010$. The data filtering criteria are the following:

- In line with the L2OS OTT, only SMOS ascending passes have been considered; in August, the galactic plane is well aligned with the descending tracks, contaminating all the SMOS scenes;

- To avoid land contamination in the image reconstruction process, the presence of land is detected at the much wider Level 1B FOV (instead of L1C). Only L1B snapshots where no single measurement is located over land are used;

- Outliers in the Alias-Free FOV, i.e., whenever the departure from forward model exceeds $20 \mathrm{~K}$ (mainly due to sea ice and probably RFI contamination), are discarded.

Note that only $11 \%$ of snapshots pass the conservative land contamination filter. The criteria applied are very strict to ensure that only the best quality measurements are considered in the computation of the OTT.

To compute $T_{B}^{\text {model }}(1)$, the theoretical Small Slope Approximation (SSA) roughness model, i.e., one of the three pre-launch roughness models used in the L2OS (see [1] for detailed references), is used. Furthermore, only images from co-polar epochs have been considered $\left(\mathrm{T}_{\mathrm{XX}}\right.$ and $\left.\mathrm{T}_{\mathrm{YY}}\right)$. Finally, the OTT statistics computed in the following sections are integrated over a circle of radius 0.3 in the antenna frame, a domain similar to the AF-FOV that allows discarding antenna pixels contaminated by the close Sky aliases.

\section{METHODOLOGY AND DISCUSSION}

To characterize the most suitable OTT, it is desirable to separate the different effects that may jeopardize the stability of the OTT. As already mentioned, the three effects under investigation are 1) the number of snapshots to be averaged, 2) their temporal and 3) their latitudinal sampling.

To study one effect at a time, the other two are fixed, so as to correctly discriminate among the different impacts. The methodology applied consists of comparing the computed OTTs in specific predefined conditions for a certain parameter (number of snapshots, temporal window width, and latitudinal coverage). By measuring the variance of the mismatches of the various OTTs, the consistency and robustness of the OTT operational algorithm is assessed.

\subsection{Impact of the number of averaged snapshots}

The OTT (Eq. 1) has random errors. These come from the measurements (e.g., thermal noise) but also the forward model and the auxiliary data used to compute the OTT (eq. 1). A straightforward way to reduce such errors is to increase the number of averaged snapshots. 
To analyze the first effect, the temporal window is set to 16 days and data available at all latitudes are considered. Note that after applying the data filtering described in Section 3, only data from $60^{\circ} \mathrm{S}$ to $40^{\circ} \mathrm{N}$ are available. The collected filtered dataset consists of roughly $\mathrm{N}=12000$ snapshots for each subset of 16 days and for each polarization.

In this experiment, the following procedure is applied. Within the constant temporal window, the total number of snapshots is randomly split (interspersed) into $n_{s}$ subsets with $\mathrm{N}_{\mathrm{s}}$ snapshots, where $\mathrm{n}_{\mathrm{s}}$ and $\mathrm{N}_{\mathrm{s}}$ range from $\mathrm{n}_{1}=60$ and $\mathrm{N}_{1}=200$ (i.e., the approximate number of snapshots used in the DPGS half-orbit OTT) to $\mathrm{n}_{17}=2$ and $\mathrm{N}_{17}=6000$ (about half of the total number of snapshots available in the filtered 16-day dataset), whereas $n_{s}$ is the largest integer verifying $n_{s} * N_{s} \leq N$. For each of the 17 subsets, $\mathrm{n}_{\mathrm{s}}$ OTTs are computed using $\mathrm{N}_{\mathrm{s}}$ snapshots each. Then, the root-mean-square (RMS) difference between each of the $\left(n_{\mathrm{s}}-1\right)$ pairs of OTTs is calculated and the $\left(\mathrm{n}_{\mathrm{s}}-1\right)$ RMS values are averaged, to have a unique value per each subset. These mean RMS values characterize the accuracy of the estimated OTT. Figure 2 shows the evolution of this mean RMS as a function of the number of snapshots used in the OTT estimation. It highlights that the collection of an increasingly larger ensemble of randomly-spread filtered snapshots in a given temporal window allows a reduction of the OTT variability. Such improvement of the OTT accuracy is in excellent agreement with the standard law of Gaussian noise reduction with number of samples. This suggests that the increase of the number of snapshots essentially leads to improve the OTT estimation accuracy by reducing the random noise. Recalling that the OTTs used at DPGS are computed from one half-orbit, i.e. about $200 \mathrm{~T}_{\mathrm{XX}}$ and $\mathrm{T}_{\mathrm{YY}}$ snapshots (i.e. RMS close to $0.25 \mathrm{~K}$ in Fig.2), the results suggest that increasing the number of snapshots in their computation could allow to increase their accuracy by $0.2 \mathrm{~K}$ RMS and by about $0.5 \mathrm{~K}$ at specific locations in the antenna frame.

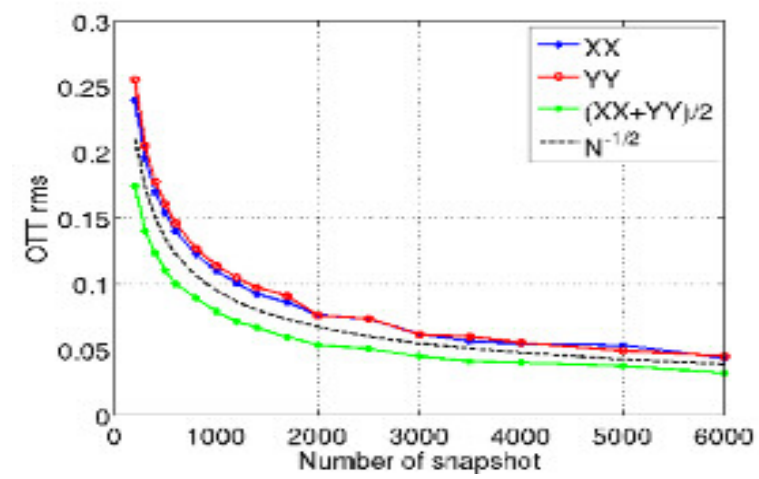

Fig. 2 Evolution of the averaged RMS of the OTT mismatches as a function of $N_{s}$, for both $T_{X X}$ and $T_{Y Y}$ and also First Stokes parameter divided by 2. Note that for each dot a different number $\left(n_{s}\right)$ of OTTs has been used to compute the averaged value.

This is verified over 3 different data types, i.e., $\mathrm{T}_{\mathrm{XX}}$, $\mathrm{T}_{\mathrm{YY}}$, and First Stokes parameter $\left(\mathrm{I}=\mathrm{T}_{\mathrm{XX}}+\mathrm{T}_{\mathrm{YY}}\right)$. Note that for First Stokes divided by 2, being the average of $\mathrm{T}_{\mathrm{XX}}$ and $\mathrm{T}_{\mathrm{YY}}$, a similar noise level is reached with only half the number of snapshots.

\subsection{Impact of the temporal variations}

The second experiment deals in turn with the OTT changes over different time periods, in order to stress the potential impact of the temporal apparent drift present in SMOS measurement, interpreted as real drift or miscalibration, according to [7].

This time, the control variables are a fixed number of snapshots and again a full latitudinal coverage. The variable parameter is, in this case, a set of 6 temporal windows of 8 days, spanning a period starting at $\mathrm{T}_{0}$ (August $3^{\text {rd }}$ ) and ending at $\mathrm{T}_{0}+48$ days (September $19^{\text {th }}$ ), based on the consideration that the OTT may change if there is a time-dependent systematic effect.

The fixed number of snapshots is defined as the minimum number of scenes available from all subsets $\left\{\mathrm{S}_{1}, \ldots, \mathrm{S}_{\mathrm{n}}\right\}$ (where $\mathrm{n}=6$ windows) after land and outlier filtering. For each subset $S_{n}$ corresponding to a temporal window width of 8 days, $N_{n}$ snapshots are available and an OTT is computed by randomly selecting a fixed number $\mathrm{N}$ of snapshots for all time windows, where $\mathrm{N}=$ $\min \left(\left\{\mathrm{N}_{1}, \ldots, \mathrm{N}_{\mathrm{n}}\right\}\right)=6250$. Note that this number $\mathrm{N}$ corresponds to a residual noise level of about $0.04 \mathrm{~K}$ and $0.05 \mathrm{~K}$ for First Stokes parameter and $\mathrm{T}_{\mathrm{XX}}$ or $\mathrm{T}_{\mathrm{YY}}$, respectively, according to Fig. 2. For all subsets, the RMS value of the difference with respect to the OTT obtained from $S_{1}$ is computed, where $S_{1}$ corresponds to the dataset obtained from the first time window, used here as the reference subset.

In Fig. 3, these average RMS values are illustrated against the final date of subset $S_{k}$, reported as the number of days since August $3^{\text {rd }}$. Shifting the temporal window from which the data are selected, the OTT does significantly change by several tenths of Kelvin, up to $0.6 \mathrm{~K}$ RMS. It has been checked that most of this RMS increase is explained by an increasing (negative) bias, while the standard deviation (SD) remains rather constant (around 0.15 and $0.2 \mathrm{~K}$ for $1^{\text {st }}$ Stokes and $\mathrm{T}_{\mathrm{XX}}$ or $\mathrm{T}_{\mathrm{YY}}$, respectively) over the entire period.

Such negative bias increases (in absolute value) at a rate of about $0.4-0.5 \mathrm{~K}$ per month. It is highly consistent with recent diagnostic on the drift induced by the Flat Target Response (FTR): several sky images were reconstructed after FTR correction and results showed that, with increasing lag between sky images and FTR acquisition dates, a negative drift of the order $0.5 \mathrm{~K}$ per month appears in the centre of the image.

With the current status of the salinity retrieval processing at DPGS, i.e. a unique OTT provided as a 
fixed auxiliary file, the present results show that 0.5-0.6 $\mathrm{K}$ discrepancy can be expected at several weeks interval, which should propagate to salinity biases of the order of 1-1.2 psu. The results also suggest that periodic OTT updates should help in reducing the SSS temporal biases. Moreover, Fig. 3 provides a useful metric to define the optimal periodicity of the OTT update.

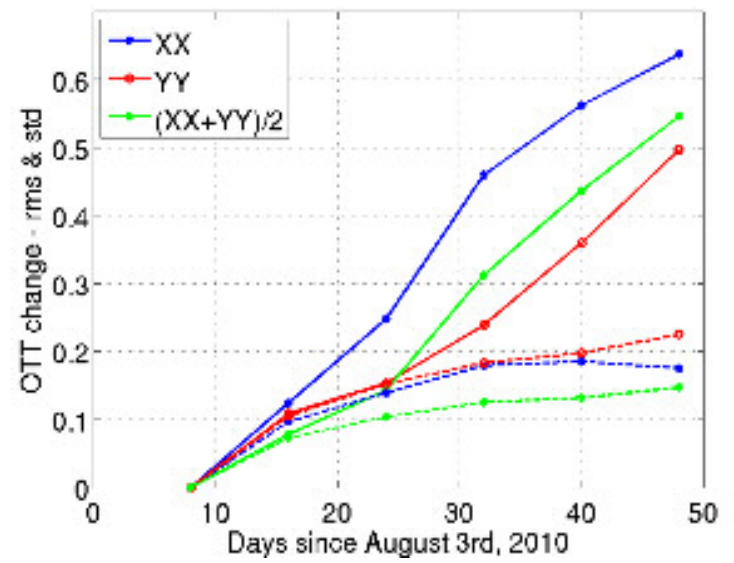

Fig. 3 Evolution of the averaged RMS of the OTT mismatches as a function of the number of days following the FTT calibration, for both $T_{X X}$ and $T_{Y Y}$, and the First Stokes parameter divided by 2. Dashed lines correspond to the averaged standard deviation.

\subsection{The Impact of latitudinal variations}

The third experiment concerns the OTT variability over different geographical areas, represented here as different latitudinal ranges, in order to evaluate the sensitivity to spatial (geographical) sampling.

The 16-days period from August $3^{\text {rd }}$ to $18^{\text {th }}$ is used, and, once filtered from land and outliers, the boresight locations of the remaining snapshots essentially span between $60^{\circ} \mathrm{S}$ and $36^{\circ} \mathrm{N}$. A reasonable trade-off between number of boxes and number of snapshot per box leads to the definition of 16 boxes of $6^{\circ}$ latitude width, i.e., $\left\{\mathrm{S}_{1}, \ldots, \mathrm{S}_{\mathrm{n}}\right\}$ subsets (where $\mathrm{n}=16$ boxes). In line with the temporal analysis, for each subset $S_{k}$ corresponding to a $6^{\circ}$ latitudinal box, $\mathrm{N}_{\mathrm{k}}$ snapshots are available and an OTT is computed by randomly selecting a fixed number $\mathrm{N}$ of snapshots for all latitudinal boxes, where $\mathrm{N}=\min \left(\left\{\mathrm{N}_{1}, \ldots, \mathrm{N}_{\mathrm{n}}\right\}\right)=610$. Note that this number $\mathrm{N}$ corresponds to a residual noise level of about $0.1 \mathrm{~K}$ and $0.15 \mathrm{~K}$ for the First Stokes parameter and $\mathrm{T}_{\mathrm{XX}}$ or $\mathrm{T}_{\mathrm{YY}}$, respectively (see fig.2). For all subsets, the RMS value of the difference with respect to the OTT obtained from $\mathrm{S}_{5}$ is computed, where $\mathrm{S}_{5}$ corresponds to the dataset from the fifth latitudinal box, centred around $36^{\circ} \mathrm{S}$ and used here as the reference subset. Note that, in principle, any box can be used as reference; we choose a somewhat centred box since the interpretation of the results is more straightforward. Also for clarity purposes, we present the results in terms of bias and SD rather than RMS.
Figure 4 displays the bias (solid) and SD (dashed) values as a function of the latitude bin center for the three modes. In line with the temporal analysis, OTT mismatches present a somewhat constant SD of about $0.2 \mathrm{~K}$ and $0.3 \mathrm{~K}$ for First Stokes and $\mathrm{T}_{\mathrm{XX}}$ or $\mathrm{T}_{\mathrm{YY}}$, respectively, except for high latitudes. These SD values are higher than those reported in temporal analysis $(0.15$ and $0.2 \mathrm{~K}$ ). This is partly explained by the different number of snapshots used in both analyses (see difference in residual noise for $\mathrm{N}=610$ and $\mathrm{N}=6250$ in Fig. 2).

Another interesting feature of Fig. 2 is the already mentioned difference in SD between First Stokes (dashed green) and $\mathrm{T}_{\mathrm{XX}}$ or $\mathrm{T}_{\mathrm{YY}}$ (dashed blue/red). The residual noise level (see fig. 2) for $\mathrm{N}=610$ is $0.1 \mathrm{~K}$ and $0.14 \mathrm{~K}$ for the First Stokes parameter and $\mathrm{T}_{\mathrm{XX}}$ or $\mathrm{T}_{\mathrm{YY}}$, respectively. By subtracting this noise level to the dashed curves of Fig. 4 (note that subtraction is performed in the variance domain, therefore over squared values of $\mathrm{SD}$ ), we would still see differences between the dashed red/blue curves and the green curve up to $0.1 \mathrm{~K}$. These higher $\mathrm{SD}$ values of $\mathrm{T}_{\mathrm{XX}}$ or $\mathrm{T}_{\mathrm{YY}}$ may be caused by misestimates of the Total Electron Content (TEC) and therefore an error in the Faraday rotation correction. The latter is known to modify the level of polarization mixing at the antenna level, resulting in opposite sign errors on $\mathrm{T}_{\mathrm{XX}}$ and $\mathrm{T}_{\mathrm{YY}}$. The First Stokes parameter is by definition unaffected by TEC. As such misestimates of TEC (or its forward model) will increase the SD of the OTT mismatches in $\mathrm{T}_{\mathrm{XX}}$ or $\mathrm{T}_{\mathrm{YY}}$, but not in the First Stokes parameter.

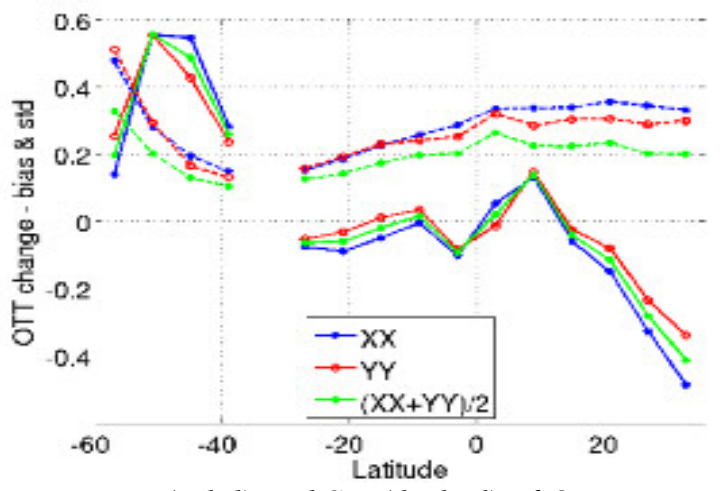

Fig. 4 Bias (solid) and SD (dashed) of OTT mismatches as a function of latitude, using OTT around $36^{\circ} \mathrm{S}$ as reference, for $T_{X X}, T_{Y Y}$ and First Stokes parameter divided by 2 .

Regarding the bias curves (solid lines) in Fig. 4, there are two distinct effects: a large positive bias at high Southern latitudes and a secondary peak around $10^{\circ} \mathrm{N}$. The former is very likely caused by inaccuracies in the forward model. Note that pre-launch surface roughness models present the largest errors at high winds, which occur very often at high latitudes. On the other hand, the secondary peak at $10^{\circ} \mathrm{N}$ appears more related to uncertainties in the auxiliary data used to model the TBs 
in Eq. 1. In this region with intense rain events, errors in the modelling of the atmospheric water content contribution to emissivity and in the associated auxiliary parameters may have a systematic component. Finally, the RMS as a function of latitude (not shown) reaches up to $0.6 \mathrm{~K}$. As for the temporal analysis, the use of a single OTT (such as in the current operational processor), results in a SSS retrieval uncertainty of about 1.2 psu. A reduction of the discussed latitudinal biases is therefore essential for a robust OTT estimation and a successful SSS retrieval.

\subsection{Residual temporal and spatial variations}

Besides the FTR-induced temporal effects, the forward model-, climatology-, and TEC-induced latitudinal effects, and radiometric noise-induced effects due to the number of snapshots used, both temporal and spatial residual variability of the order of $0.15-0.25 \mathrm{~K}$ still remains unexplained. Intrinsic instrument stability tests show that the latter cannot be caused by the instrument itself. Several possible factors have been identified during and after IOCP and might contribute to these residual errors having spatio-temporal structure. Among them, residual Sun-induced heating/cooling effects may be poorly accounted for in the calibration procedure, due to missing physical temperature estimates in some receptor antenna model. Also, the very bright Sun is often present in the far-from-boresight domain of the observed scenes and a correction is applied to cancel its signature; preliminary results have shown that this correction may induce residual biases of the order of $1 \mathrm{~K}$ in the near-boresight domain. Finally, foreign sources such as reflected Sun and celestial signals may also contaminate the observed scene. Regardless of whether these contributions are poorly (celestial glint) or not (Sun glint) accounted for in the forward model, both their temporal and latitudinal variations may be propagated to the OTT estimate.

\section{CONCLUSIONS}

Systematic instrumental and reconstruction errors present in SMOS images substantially degrade the SSS retrievals and must therefore be removed or at least mitigated. The so-called OTT technique is used for this purpose. In this study, a number of experiments have been designed to characterize the accuracy and the undesired variability of the OTT.

The first experiment has shown that estimating the OTT with 6000 snapshots instead of 200 snapshots (current operational setting) results in a reduction of OTT inaccuracies (random errors) of about $0.2 \mathrm{~K}$, for both the $\mathrm{T}_{\mathrm{XX}}$ and $\mathrm{T}_{\mathrm{YY}}$. The second and third experiments demonstrate that spatial and temporal data variability induces inconsistencies of up to $0.6 \mathrm{~K}$. Such inconsistencies are expected to propagate down to retrieved salinities and result in large spatio-temporal random and systematic errors.
Aiming at reducing the instrument drift-induced temporal biases in the currently retrieved salinities, a periodic upgrade of the OTT may be considered, until calibration and processing strategies implemented in the operational chain reach an adequate accuracy level. These experiments provide useful insights to set the periodicity of OTT updates by defining an appropriate trade-off between minimizing the inaccuracies shown in Figure 3, and accounting for noise reduction as described in Figure 2.

Furthermore, this work highlights the importance of accounting for (either by correcting, modelling, or filtering) the various error sources (Sun, galaxy, TEC, etc.), which affect SMOS $\mathrm{T}_{\mathrm{B}}$ measurements prior to OTT computation and SSS retrievals.

Finally, forward model inaccuracies, which can vary from model to model, have a substantial impact in the OTT computation. Further activities to optimize the stability of the OTT are matter of ongoing research [5], [8].

\section{ACKNOWLEDGEMENTS}

This work was supported by the Spanish National R+D Plan for the SMOS Barcelona Expert Center on Radiometric Calibration and Ocean Salinity activities, through project MIDAS-6 AYA2010-22062-C05 and previous grants.

\section{REFERENCES}

[1] Font, J. et al., "SMOS: The challenging sea surface salinity measurement from space," Proc. IEEE, vol. 98, pp. 649-665, 2010.

[2] McMullan, K.D. et al., "SMOS: The Payload," IEEE Trans. Geosci. Remote Sens., 46 (3), pp. 594-605, 2008.

[3] Zine, S. et al., "Overview of the SMOS Sea Surface Salinity Prototype Processor," IEEE Trans. Geosc. Remote Sens, 46 (3), 621-645, 2008.

[4] Camps, A. et al, "Retrieving Sea Surface Salinity with Multi-angular L-band Brightness Temperatures: Improvement by Spatio-temporal Averaging", Radio Sci., 40 (2), 2005.

[5] Talone, M. et al., "SMOS' brightness temperatures statistical characterization", IGARSS 2010 proceedings, Honolulu, HA, USA, 25-30 July 2010.

[6] Font J. et al., "SMOS first data analysis for sea surface salinity determination", accepted in Int. J. Rem. Sens., 2011.

[7] Tenerelli, J., N. Reul, "SMOS Brightness Temperatures over the Global Oceans: Contaminating Effects and Salinity Retrieval", In Proceedings ESA Living Planet Symposium, 28 June-2 July 2010, Bergen, Norway, ESA SP-686.

[8] Torres, F. et al., "Minimization of image distortion in SMOS brightness temperature maps over the ocean", IEEE Geosc. Rem. Sens. Lett., in press, 2011. 